\title{
METRICS FOR OBJECTIVE ONTOLOGY EVALUATIONS
}

\author{
Robert J. Pefferly Jr. \\ Sunitia, Tallinn, Estonia, Tel:+372 5147 099, http://www.sunitia.com/ \\ rob@sunitia.com
}

Michael C. Jaeger

TU Berlin, SEK FR6-10, Franklinstrasse 28/29, 10587 Berlin, Germany

mcj@cs.tu-berlin.de

Moussa Lo

UFR de Sciences Appliquees et de Technologie, Universite Gaston Berger, BP 234 Saint-Louis, Senegal, Tel:+221 9612340 , http://www.ugb.sn/

lom@ugb.sn

\begin{abstract}
We present new metrics and techniques which allow one to configure a metadata catalogue and objectively describe knowledge management ontologies. Per C.E. Shannon (1948), when describing information based systems, statistical measures are a necessity; yet very few ontology based standards mention quantifiable measures such as entropy, data encapsulation, complexity, efficiency, evolution, or redundancy. We hope to demonstrate how statistical information measures can be implemented for ontology-based knowledge management systems using our $\mathbb{L}_{0}$ statistic, entropy, evolution, organization, sensitivity, and an interpretation of complexity.
\end{abstract}

\section{Introduction}

As demonstrated by Shannon, 1948, when evaluating information based systems, statistical measures are a necessity, yet unfortunately most popular management 'standards' that exist today rely too much on the subjective world of 'business processes' and not enough on consistent mathematical foundation principles. For examples, refer to Magkanaraki et al., 2000, Li et al., 2003, the Process Interchange Format, and Framework (PIF) and Workflow Management Coalition (WfMC) specifications. Thus, current knowledge manage- 
ment standards are inconsistent by definition and doomed to failure in large scale heterogeneous interactions. This emphasis on subjective 'business processes,' and lack of quantitative standards/measures has created a major stumbling block for the Business Process, Knowledge Management, Data Libraries, and Semantic Ontology fields.

Even though a great deal of effort has been spent on developing standards, as demonstrated in Magkanaraki et al., 2000 and the Santa Fe Institute work, even when objective measures are utilised, it is common to use 'hard number' evaluations when describing probabilistic 'soft number' informatic entities.

\begin{abstract}
For several reasons, the development of real-world enterprise-wide knowledge managements *ontology-based knowledge management systems* is still in the early stages. First, despite much research on ontology representations, engineering, and reasoning, features such as scalability, persistancy, reliability, and transactions - standardized and widely adopted in classical database-driven information systems - are typically not available in ontology-based systems. Maedche et al., 2003
\end{abstract}

The true innovation of this paper lies in the informatic metrics we prescribe for describing/comparing complex ontology based systems. We hope to demonstrate how objective statistical information measures can be implemented for objective knowledge management - one can now compare ontologies and answer the question: 'Is one ontology better than another?'

\title{
1.1 Background
}

Remark 1. An ontology is a collection of symbols used to express data. Although ontologies possibly cover extremely technical fields or address complicated expertise, they are structurally nothing more complex than a finite set of symbols with a bijective mapping.

In knowledge management systems, there are a number of 'big picture' issues that need to be explored, elaborated, and corrected since there is a disconnection between the popular 'Management Science' literature surrounding knowledge management and the underlying informatic principles. The emphasis on building 'Unambiguous Semantics' and 'Light-weight Inferences' such that ontologies have meaning to a human reader is a nice by product, but is immaterial and moot from an informatic perspective; it is the Shannon Information (i.e. Entropy $\mathcal{H}$ ) that is important. For example, whether we call a rose a 'roza,' 'roos,' 'fleur rouge, 'red.flower,' 'Mary's favourite,' ' $\Omega$,' or 'H1026' - it is still a rose.

Remark 2. For a practical example of an ontology, refer to the Information Ontology Root Li et al., 2003. Motik et al., 2002 presents a starting point for the practical questions: 'What is an ontology?' and 'How does one create an knowledge model?' 
There will always be variability and questionable assumptions when modeling physical problems, hence we are solely concerned with knowledge encapsulation to maximize the sufficiency of the metadata. In order to do this, not only does one have to capture a given degree of granularity, but one must also address how complex/efficient a system is using an respective ontology. Hence, entropy related measures provide the foundation for information metrics. Thus, the crux of any knowledge system is the information content and knowledge encapsulation that maximizes the sufficiency of the metadata; not in whether an ontology is subjectively 'linguistically correct' or follows 'common sense.'

First, we realize that imposing a single ontology on the enterprise is difficult if not impossible. Maedche et al., 2003

Motik et al., 2002 attempts to put this into light with an objective view of ontology formulations, but once again forces systems to bend to a inconsistent world. Their argument on Light-weight Inferences is a representation of redundancy that aids a modeler in understanding the system such that she can envision how to make a system scalable and tractable. Organizations have not been able to break this a priori mindset as demonstrated by the standards that have been established; WFMC, PIF, and other ontology standards assume a general model that is built on business practices, this is fundamentally flawed. We argue that if the paradigm of the a priori data base is truly cast aside, then by using a Shannon Information approach, the system will be both scalable and tractable as well as efficient. This requires a complete point-of-view change from the programmer/developer that seems to be lacking in most ontology based designs - we continue to fight disorder and chaos instead of utilize it as a informatic tool.

\subsection{Why are objective metrics necessary?}

... the life cycle of ontology design can be summarised as three major stages, i.e. building, manipulating and maintaining. ... During developing the proposed system, establishing definite and consistent ontology is perhaps the toughest task. ... In this study, one year was spent to establish a common consensus and then to define the initial domain ontology in the metal industry. Li et al., 2003

This is not an uncommon occurrence where an 'inordinate amount of time' is spent on a 'repetitive and arduous task,' thus hindering development, management acceptance, and acceptability of knowledge management. Knowledge Engineers must justify this 'waste of time and resources' to management since current industrial standard ontologies are often not applicable to organizations without major modifications. A break from subjective evaluations to objective metrics would alleviate biased opinions and allow one to describe an ontology. Using appropriate metrics one can address building stage issues such as: 


\section{Building Stage:}

- Instead of spending one year of a project developing an ontology, maybe only six months was substantially beneficial for effective information content. Points of diminishing returns can be identified and resources reallocated appropriately.

- One can finalize an iterative building process once the recommended changes add less than ' $\mathrm{X}$ ' value to the knowledge management.

\section{Manipulating Stage:}

- The question of 'Is one ontology more effective than another?' can be ob jectively evaluated.

- How does one determine if the 'coverage' of an ontology is sufficient?

- What is the value added when changing elements in an ontology?

\section{Maintaining Stage:}

- Questions such as 'Is one ontology easier to maintain than another?' can be addressed.

- What does the addition or elimination of term ' $\mathrm{X}$ ' do to the system? Will there be unintended consequences?

General: An iteration of domain expert solicitation must occur, but it is difficult to measure progress, hence, organizations must address issues such as:

- What is the value added with each iteration?

- When manipulating ontologies, how does one demonstrate positive improvement?

- When changing the terminology or structure of an ontology, how does one demonstrate the change is either beneficial, detrimental, or an exercise in futility?

- When maintaining an ontology, when does the redundancy and complexity become prohibitive?

\subsection{Shannon discrete source model}

Communication is simply a process of exchanging information via a channel encompassing \{ human - human, human - computer, computer - human, computer - computer $\}$ interactions It is hard to identify any information paradigm that does not follow a Shannon model where: 
- Any phenomena which send discretized data via a finite set of packets is considered a Shannon discrete source and can be modeled via a Markov chain.

- The symbology of an ontology is secondary to the information that it encapsulates, thus, we are concerned with a series of symbols being used to represent data and the information that is passed via the symbolic chain.

- Whether data is transmitted as binary data packets, IP streams, words, smoke signals, or sounds, it is difficult to identify an ontology information paradigm that does not follow a Shannon model

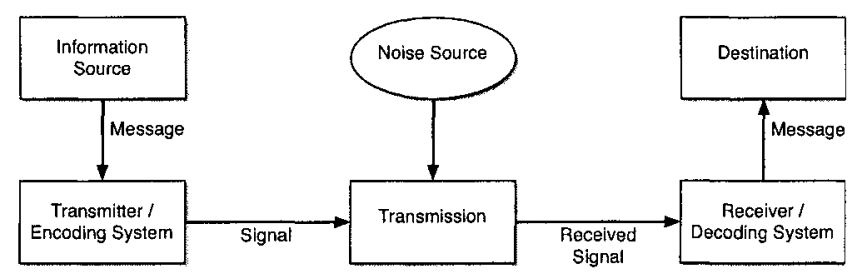

Figure 1. Shannon Model - Schematic diagram of a general communication system

\section{Data, information, and metrics}

Data is a hard number raw output of a discretized process represented by a countable set of symbols. Information is a level of abstraction above data, where data is a finite set of symbols such that the order of the symbols may contain 'meaning' - i.e. information as a sufficient statistic of a data set relative to a query.

For the purposes of this chapter we use the term 'information' to refer to a spatiotemporal pattern that can be understood and described independently of its physical realization. Stephanie Forrest, 2000, page 362

Thus, one searches processed data to derive soft number information, where information is only 'meaningful' if it appropriately answers a query using an appropriate data set. The concept of data versus information is lost in most knowledge system literature; i.e. 'information' should be replaced with 'data.'

Enterprises don't lack for information: they are drowning in it. So knowledge workers need all the help they can get in separating the wheat from the chaff. Savage, 2003 
Second, a large body of information in an enterprise typically already exists outside the knowledge manage system - for example, in other applications such as groupware, databases, and file systems. Motik et al., 2002

Definition 1. A hard number is a weighted singularity, such that it is a deterministic point estimate with 0 variance. Thus the expected value is $\mathbb{E}($ Hard Number $)=\alpha$ and the variance is $\mathbb{V}($ Hard Number $)=0$.

Definition 2. A soft number is a stochastic point estimate where $\mathbb{E}($ Soft Number $)=\alpha$ and $\mathbb{V}($ Soft Number $) \neq 0$

Data is measured in hard numbers while soft numbers represent 'fuzzy' information values that do not have a tangible representation. For example, when looking at a picture, one does not individually observe every pixel (data) on a screen in strict numeric order to ascertain what the picture conveys (information). Thus, soft numbers are a sufficient description involving probability, expectations, and variances. Hence, when discussing hard numbers, $1+2=3$ without question. For soft numbers, $1+2 \neq 3$ is almost surely true, but given certain restrictions, $\mathbb{E}(1)+\mathbb{E}(2)=\mathbb{E}(3)$ may be true. Although the difference may seem as a 'techy' pet peeve, the implication for designing knowledge management systems are quite profound.

\subsection{Complexity is not information}

There a difference between statistical complexity and computational complexity and the common use of statistical complexity being referred to as a Shannon measurement equivalent to entropy is incorrect. Further, the common use of 'statistical complexity' being referred to as a Shannon measurement equivalent to entropy is incorrect. The following as an excellent example of the disconnection between complexity and entropy.

It is emphasised that, given an entropy value, there are many possible complexity values, and vice versa; that is, the relationship between complexity and entropy is not one-to-one, but rather many-to-one or one-to-many. It is also emphasised that there are structure in the complexity-versus-entropy plots, and these structures depend on the details of a Markov chain or a regular language grammar. Li, 1991, page 381

\subsection{New metrics}

The following metrics were inspired by Hilderbrand, 1968 and further enhance the concepts presented in Martin Hepp, 2005, Section 2.

Definition 3. The computational complexity of a system $F$ will be defined in terms of a non-standard. $\beta=\mathrm{O}(g(x))$, where $\beta$ is 'Big-Oh' with respect to $g(x)$, when $\lim \frac{|\beta|}{g(x)} \leq K$ almost surely. 
Computational complexity is sometimes referred to as Kolmogorov complexity, Gacs, 2001 but we will utilize a non-standard Big-Oh notation where the magnitude of $\mathrm{K}$ will be of importance.

Definition 4. The $\mathbb{L}_{0}$ statistical complexity is defined by the inverse of the following expectation where card $(\mho)$ is the cardinality of the domain space:

$$
\mathbb{L}_{0}\left(F_{N}(x)\right)=\mathbb{E}\left[\sqrt{\frac{\mathbb{P}(x)}{\operatorname{card}(\mho)}}\right]^{-1}=\frac{1}{\sum_{j=1}^{N} \mathbb{P}\left(x_{j}\right) \sqrt{\frac{\mathbb{P}\left(x_{j}\right)}{\operatorname{card}(\mho)}}}
$$

As discussed in Shannon, 1948 a uniform distribution is optimal for information content, thus our $\mathbb{L}_{0}$ statistic is based on the Discrete Uniform Probability Distribution and has a lower bound of 0 with an unlimited upper bound. For example, an equiprobably coin has a $\mathbb{L}_{0}$ value of 2 and a 6 sided equiprobably die has a $\mathbb{L}_{0}$ value of 6 .

Definition 5.The organization or structure of a process will be

$$
\Phi\left(F_{N}(x)\right)=\mathbb{E}(\mathbb{P}(x))=\sum_{j=1}^{N} \mathbb{P}^{2}\left(x_{j}\right)
$$

There is a definite need to distinguish between organization and complexity but most authors assume a limited view on how 'correlation typically provides a lower bound of a measure of complexity.' Organized structures are independent of both the entropy and complexity, thus a separate metric $\Phi$, is a necessity.

Definition 6. The evolution of a process will be $\mathcal{E}(F(x))=\Phi(F(x))^{-1}$. A simple structure indicates that a system is highly organized and the more organized a system is, the smaller its evolution. The difference between two ontology evolution values represent a measure of informatic distance. To draw an analogy: physical mass is measured in grams and distance in meters; information 'mass' is measured in $\mathbb{L}_{0}$ units and the distance is measured using evolution.

Definition 7. The sensitivity is a first order difference (change) in the evolution of a system when an element is either eliminated (or added) from the process. Such that $\mathcal{S}\left(F_{N}(x)\right)=\Delta \mathcal{E}\left(F_{N}(x)\right)=\mathcal{E}\left(F_{N \pm 1}(x)\right)-\mathcal{E}\left(F_{N}(x)\right)$, where $\operatorname{card}\left(F_{N}\right)=N$ and $\operatorname{card}\left(F_{N \pm 1}\right)=N \pm 1$.

Sensitivity will be a measure similar to the sensitivity of a numerical approximation, in that it will be a measure of the effect a small change in the structure 
has on the overall system. For our purposes, sensitivity will be measured as a finite difference of the organization metric via the addition/deletion of a term.

\subsection{Standard Metrics}

The following metrics are standard definitions as defined by Shannon, 1948:

Definition 8. The entropy $\mathcal{H}$ of a system will be defined as:

$$
\mathcal{H}\left(F_{N}(x)\right)=\mathbb{E}(-\log (\mathbb{P}(x)))=-C \sum_{j=1}^{N} \mathbb{P}\left(x_{j}\right) \log \left(\mathbb{P}\left(x_{j}\right)\right)
$$

per Shannon, 1948, Theorem 2. The choice of $C$ is merely a normalizing correction for the a unit measurement.

Notation 9. Relative entropy is the percentage of entropy realized with a coding system as compared to the Shannon theoretic value for the maximal entropy of a system. The relative entropy of a system $F$ will be defined as $\mathcal{H}_{\text {Rel }}\left(F_{N}\right)=\frac{\mathcal{H}_{\text {practical }}\left(F_{N}\right)}{\mathcal{H}_{\text {theoretical }}\left(F_{N}\right)}$.

Relative entropy is the percentage of entropy realized with a coding system as compared to the Shannon theoretic value for the maximal entropy of a system.

Notation 10. The redundancy of a system $F$ will be defined as $\mathcal{R}=1-$ $\mathcal{H}_{\text {rel }}\left(F_{N}\right)$.

\subsection{Practical Examples}

The following Table 1 indicates the relative metrics for the examples of this section. The sensitivity is calculated by removing the first element.

\begin{tabular}{|l|l|l|l|l|}
\hline Metric & Theoretical die & Bayesian die & A priori ontology & Bayesian ontology \\
\hline $\mathcal{H}$ & 0.778 & 0.736 & 0.7009 & 0.714 \\
\hline $\mathcal{H}_{\text {Rel }}$ & 1 & 0.946 & 0.9007 & 0.9176 \\
\hline $\mathcal{R}$ & 0 & 0.0536 & 0.0993 & 0.0824 \\
\hline$O$ & 6 & 6 & 6 & 6 \\
\hline $\mathbb{L}_{0}$ & 6 & 5.592 & 5.334 & 5.442 \\
\hline$\Phi$ & 0.1667 & 0.2 & 0.22 & 0.2099 \\
\hline $\mathcal{E}$ & 6 & 5 & 4.545 & 4.765 \\
\hline $\mathcal{S}$ & -1 & -0.736 & -0.396 & -0.451 \\
\hline
\end{tabular}

Table 1. Metric results 
A six sided die - Theoretical. For a relatively easy example, assume that one is trying to describe the physical phenomena of rolling a six sided equiprobable die. The die operates in a discrete space where each side of the die has a one in six chance of appearing, therefore, the probability density function (PDF) $\mathfrak{f} \sim \mathcal{U}\left(\frac{1}{6}\right), \mathbb{E}(\alpha)=3 \frac{1}{2}, \mathbb{V}(\alpha)=2 \frac{11}{12}$. Notice that this is information, how often does one role a six sided die and end up with $3 \frac{1}{2}$ ?

A six sided die - Bayesian. Assuming that the six sided die is 'too complex' for one to understand, roll the die $N=10$ times and plot the histogram of the data. Assuming that a six sided die $\widehat{F}$ is rolled, the realization of a the result of the die will consist of an iid random data set is $\{3,1,6,6,4,5,2,3,6,5\}$. Using this information one can estimate that: $\widehat{\mu}_{\alpha}=4.1$ and $\widehat{\sigma}_{\alpha}^{2}=3.211$.

Theoretical Ontology. A six term ontology is designed by a knowledge engineer who estimates a priori probability values for filling data elements per values outlined in Table 2 .

\begin{tabular}{|l|c|}
\hline Element $(x)$ & $\mathbb{P}(x)$ \\
\hline Version & $\frac{1}{20}$ \\
\hline Summary & $\frac{1}{20}$ \\
\hline Organization & $\frac{3}{20}$ \\
\hline Date & $\frac{4}{20}$ \\
\hline Author & $\frac{5}{20}$ \\
\hline Title & $\frac{6}{20}$ \\
\hline
\end{tabular}

Table 2. A priori ontology

Practical Ontology. The same ontology is utilized where Bayesian a posterior probabilities are derived from 'real instances' stored in the catalogue; as shown in Table 3. In this table, five instances of a model are utilized
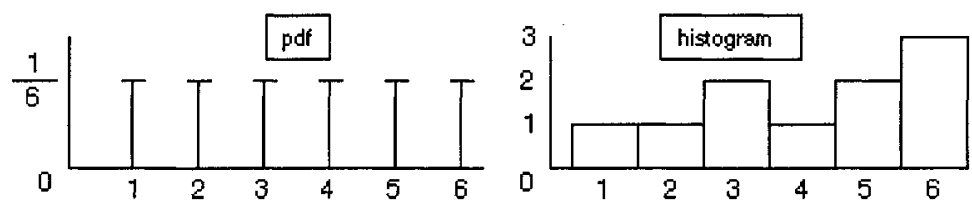

Figure 2. PDF versus histogram 
$M 1, M 2, M 3, M 4, M 5$ where an $\mathrm{X}$ implies that the data entry is filled for that instance.

\begin{tabular}{|l|l|l|l|l|l|l|}
\hline Element $(x)$ & $\mathrm{M} 1$ & $\mathrm{M} 2$ & $\mathrm{M} 3$ & $\mathrm{M} 4$ & $\mathrm{M} 5$ & $\mathrm{P}(x)$ \\
\hline Version & & $\mathrm{X}$ & & & & $\frac{1}{18}$ \\
\hline Summary & & & & $\mathrm{X}$ & & $\frac{1}{18}$ \\
\hline Organization & $\mathrm{X}$ & $\mathrm{X}$ & & $\mathrm{X}$ & & $\frac{3}{18}$ \\
\hline Date & $\mathrm{X}$ & $\mathrm{X}$ & $\mathrm{X}$ & $\mathrm{X}$ & & $\frac{4}{18}$ \\
\hline Author & $\mathrm{X}$ & & $\mathrm{X}$ & $\mathrm{X}$ & $\mathrm{X}$ & $\frac{4}{18}$ \\
\hline Title & $\mathrm{X}$ & $\mathrm{X}$ & $\mathrm{X}$ & $\mathrm{X}$ & $\mathrm{X}$ & $\frac{5}{18}$ \\
\hline
\end{tabular}

Table 3. A posterior ontology

\section{Conclusion}

In this paper we postulate that there is a distinct disconnection between complexity, entropy, and the structure/organization of an ontology, where a lack of objective metrics is the crux of the knowledge management problem. We hope that others will question our 'new' measures and independently vindicate or vilify our constructs. We stress that our metrics warrant independent verification.

Ontology management is not a trivial matter since knowledge management users delete/add terminology, data elements and their inter-dependencies change

Changing an ontology can induce inconsistencies in other parts of the ontology. Semantic inconsistency arises if an ontology entity's meaning changes. (...) An ontology update might also corrupt ontologies that depend on the modified ontology and, consequently, all artefacts based on these ontologies. (...) However, apart from syntax inconsistency, semantic inconsistency can also arise when, for example, the dependent ontology already contains a concept that is added to the original ontology. Maedche et al., 2003

Hence, there is an justifiable need for objective metrics such that different knowledge management ontologies can be compared on equal footing. This is still a very subjective field when objectivity should be the goal of the discipline in order to remove the subjective nature surrounding knowledge, thus minimizing miscommunication. By establishing objective measures implementing metadata metrics, solid definitions will improve the overall state of the knowledge management field - if you can measure it, you can manage it.

\section{References}

Stephanie Forrest, Steven A. Hofmeyr, Immunology as Information Processing, Oxford University Press, England, 2000, Eds. L.A. Segel and I.R. Cohen, Design Principles for Immune System \& Other Distributed Autonomous Systems, 
Peter Gacs, Quantum Algorithmic Entropy, J. Phys. A: Math. Gen. 34 (2001), 6859-6880, June 2001

Martin Hepp, Joerg Leukel, Volker Schmitz, Content Metrics for Products and Services Categorization Standards, Proceedings of the 2005 IEEE International Conference on e-Technology, e-Commerce and e-Service, pages 740-745, April 2005

Francis B. Hilderbrand, Finite-Difference Equations and Simulations, Prentice-Hall, Englewood Cliffs (NJ), 1968

Sheng-Tun Li and Huang-Chih Hsieh and I-Wei Sun, An Ontology-based Knowledge Management System for the Metal Industry, The Twelfth International World Wide Web Conference (WWW'03), Budapest, Hungary, May 2003

Alexander Maedche and Boris Motik and Lijiljana Stojanovic and Rudi Struder and Raphael Volz, Ontologies for Enterprise Knowledge Management, IEEE Intelighent Systems, pages 26-33, Volume 18, Number 2, 2003

Aimilia Magkanaraki and Sofia Alexaki and Vassilis Christophides and Dimitris Plexousakis, Benchmarking RDF Schemas for the Semantic Web, Institute of Computer Science, FORTH, Heraklion, Greece, EU Project OntoWeb and Question-How, Volume 6, 2000

Boris Motik and Alexander Maedche and Raphael Volz, A Conceptual Modeling Approach for Semantis-Driven Enterpise Applications, Springer - (ODBASE-2002), California http://kaon.semanticweb.org/papers, 2002

Peter R. Savage, The Data Alchemists, IEEE Spectrum Online, July 2003

Claude E. Shannon, A Mathematical Theory of Communication, The Bell System Technical Journal, pages 379-423, Volume 17, No. 3, 1948

Wentian Li, On the Relationship Between Complexity and Entropy for Markov Chains and Regular Languages, Complex Systems, pages 381-399, Volume 5, No. 4, 1991 